\title{
Estudio de Internacionalización a América Latina de un Software basado en la nube
}

\section{Internationalization Study to Latin America of a Cloud-based Software}

\author{
Dña. Jessica Romero Guzmán \\ Departamento de Informática Universidad \\ Carlos III de Madrid \\ Madrid, Spain \\ jeromero@inf.uc3m.es
}

Phd D. Ángel García-Crespo

Profesor Phd y Director del Instituto Pedro Juan

de Lastanosa

Universidad Carlos III de Madrid

Madrid, Spain

acrespo@ia.uc3m.es

\author{
Phd Dña. Inmaculada Puebla Sánchez \\ Profesora Phd. y Directora del Centro de \\ Simulación Empresarial. FCJYE \\ Universidad Francisco de Vitoria \\ Vicepresidenta $1^{\text {a }}$ SESGE \\ Madrid, Spain \\ i.puebla.prof@ufv.es
}

Phd. D. Ignacio Temiño Aguirre

Profesor Phd. UFV y Secretario General y CEO de CESGAR (Confederación Española de

Sociedades de Garantía Recíproca)

Universidad Francisco de Vitoria Madrid, Spain

i.termino.prof@ufv.es

Resumen - Este trabajo que presentamos enfoca la importancia de integración entre diferentes sistemas y la percepción del éxito de internacionalización de un software. Los sistemas informáticos son diseñados y utilizados por los seres humanos como lo indica el estudio "Using the Affect Grid to Measure Emotions in Software Requirements Engineering” [1]. El conocimiento de múltiples fuentes y el desarrollo eficiente de un proyecto supone tiempo y coste convirtiéndose en un modelo a seguir. Para la internacionalización del software a presentar se harán estimaciones donde el estudio será enfocado principalmente entre dos tipos de cambios regulatorios y técnicas que impliquen un desarrollo eficiente del proyecto llevando a cabo una dinámica de sistemas; Jay W. Forrester insiste que los modelos deben formar parte de un proceso de comunicación más persuasivo y que el tiempo para explotar la naturaleza dinámica de los sistemas sociales deben utilizar el conocimiento resultante para mejorar las instrucciones y las economías [2]. MiFID II (Markets in Financial Instruments Directive II) y Dodd-Frank Act (Ley de Reforma de Wall Street y Protección al Consumidor Dodd-Frank) son los nombres de los cambios regulatorios que acelerarán el crecimiento de un mercado global [3]. Estas regulaciones tienen una influencia directa sobre Europa y Norteamérica, extendiéndose a casi todo el sector financiero de forma global. Se espera que el mercado crezca a un 22,02\% de tasa anual compuesta de crecimiento (CAGR), alcanzando los \$3.040 millones [4]. Dentro de este mercado, la empresa a estudiar permite grabar y cifrar conversaciones de teléfonos fijos, móviles, correos electrónicos, faxes y SMS, entre otros. Se ha seleccionado para el estudio LATAM (Latino América), por estrategia corporativa del modelo de empresa a crear. Se ha estudiado para este modelo cuentas variables económicas de crecimiento del PIB real, PIB per cápita, entre otros indicadores como riesgo país o doing business. El resultado ponderado unido al tamaño de mercado de telefonía muestra que los países con mayor potencial a estudiar sean México y Brasil, representando el $53 \%$ del total de mercado Latinoamericano. La relación financiera entre México, Europa y Estados Unidos unido a la Ley Federal de 
Telecomunicaciones y Radiodifusión que obliga a las operadoras a grabar las llamadas de teléfono y guardar los registros durante 5 años hacen que sea México el país seleccionado para la internacionalización de la empresa. Se busca que el método de entrada sea por venta directa, logrando mantener un control tecnológico del producto y evitando un riesgo en copia de la tecnología. Es clave controlar los costes y que sean principalmente variables, de tal forma que se pueda mejorar el payback a menos de dos años, reduciendo el riesgo propio de las soluciones tecnológicas a quedar obsoletas.

Palabras clave- Internacionalización, Cloud (Nube), grabaciones, integración, BFSI, MiFID II, líneas móviles, líneas fijas, LATAM.

Abstract - This paper focuses on the importance of the integration between different systems and the perception of the success of internationalization of a software. Computer systems are designed and used by human beings as indicated in the study "Using the Affect Grid to Measure Emotions in Software Requirements Engineering" [1]. The knowledge from multiple sources and the efficient development of a project involves time and cost becoming a model to follow. For the internationalization of the software to be presented, estimates will be made where the study will be focused mainly between two types of regulatory and technical changes that imply an efficient development of the project carrying out a systems dynamics study; Jay W. Forrester insists that the models must be part of a more persuasive communication process and that the time to exploit the dynamic nature of social systems must use the resulting knowledge to improve instructions and economies [2]. MiFID II (Markets in Financial Instruments Directive II) and the Dodd-Frank Act are the names of the regulatory changes that will accelerate the growth of a global market [3]. These regulations have a direct influence on Europe and North America, extending to almost the entire financial sector globally. The market is expected to grow at a $\mathbf{2 2 . 0 2 \%}$ compound annual growth rate (CAGR), reaching \$3.04 billion [4]. Within this market, the company to be studied allows recording and encrypting conversations of fixed telephones, mobiles, e-mails, faxes and SMS, among others. LATAM (Latin America) has been selected for the study, due to the corporate strategy of the business model to be created. Economic growth variables of real GDP, GDP per capita, among other indicators such as country risk or doing business have been studied for this model. The weighted result together with the size of the telephony market shows that the countries with the greatest potential to be studied are Mexico and Brazil, representing $53 \%$ of the total Latin American market. The financial relationship between Mexico, Europe and the United States, together with the Federal Law of Telecommunications and Broadcasting, which obliges operators to record telephone calls and keep records for 5 years, make Mexico the country selected for the company's internationalization.

It is sought that the method of entry is by direct sale, achieving to maintain a technological control of the product and avoiding a risk in copy of the technology. It is key to control costs and that they are mainly variable, in such a way that the payback can be improved in less than two years, reducing the own risk of the technological solutions to become obsolete.

Keywords- Internationalization, Cloud, recordings, integration, BFSI, MiFID II, mobile lines, fixed lines, LATAM.

\section{Análisis de situación}

El modelo de empresa creado engloba la consultoría tecnológica y digital dedicada al desarrollo de aplicaciones Sofware-as-a-Service y soluciones basadas en la nube, con más de 20 años de experiencia en el mercado. Los servicios ofrecidos están enfocados en el área de voz y datos, arquitecturas IT complejas, analítica y AI. Alguno de los productos más destacados son los servicios de grabación de llamadas en la nube, y eComFax, una plataforma de Fax virtual diseñada para la movilidad e integración con e-mail; además el modelo posee servicios de grabaciones de llamadas e interacciones convergentes que permite grabas conversaciones de teléfonos fijos y móviles así como también permite la grabación de correos electrónicos, faxes y SMS, todas estas son capturadas, grabadas y cifradas de manera segura permaneciendo almacenadas en servidores y a disponibilidad del cliente en todo momento y durante un largo periodo de tiempo, siendo un servicio adaptable tanto para PyMES como para grandes empresas.

Entre las principales funcionalidades del producto ofrecido se destacan:
1. Grabación de llamadas a través de la integración con telcos,

2. Grabación de llamadas en línea fijas y líneas móviles,

3. Almacenamiento a largo plazo de llamadas para cumplimiento de las directivas de seguridad (+5 años),

4. Transcripciones automáticas de llamadas con la opción de aplicar análisis de texto para la generación de data de valor,

5. Trazabilidad de las llamadas grabadas y sus detalles para cumplir con GDPR (General Data Protection Regulation).

Las funciones de trazabilidad y almacenamiento de datos a largo plazo le permite al modelo de empresa entrar en cumplimiento con las regulaciones de seguridad implementadas por la Unión Europea GDPR [5], Markets in Financial Instruments Directive MiFID II [3] y Payment Card Indrustry Data Security Srandar PCI-DSS [6].

La cobertura de líneas fijas y de líneas móviles es diferente para cada una; para las líneas fijas la grabación de 
llamadas es un servicio que se considera propio, y para las líneas móviles se necesitará de los telcos como intermediario, por lo que, para este caso, si una empresa quiere contratar este servicio, será necesario a través de esta integración.

\section{Análisis VRIO}

El análisis realizado muestra que el modelo de la empresa a implementar dispone de recursos tecnológicos, humanos y financieros sostenibles a largo plazo que le permiten desarrollar ventajas competitivas en el mercado.

Tabla 1. Análisis VRIO. Fuente: propia.

\begin{tabular}{|c|c|c|c|c|c|}
\hline & Valuable & Rare & $\begin{array}{l}\text { Costly to } \\
\text { imitate }\end{array}$ & $\begin{array}{l}\text { Organized to } \\
\text { capture value }\end{array}$ & $\begin{array}{c}\text { Sustainable } \\
\text { C.A. }\end{array}$ \\
\hline \multicolumn{6}{|c|}{ Tangible } \\
\hline $\begin{array}{l}\text { Physical } \\
\text { Oficinas, Los servicios en la } \\
\text { nube implican pocos recursos } \\
\text { fisicos, lo que les da una } \\
\text { ventaja }\end{array}$ & Si & No & - & . & - \\
\hline $\begin{array}{l}\text { Financial } \\
\text { Bajos costes de producción y } \\
\text { suficientes fondos propios. }\end{array}$ & si & No & . & . & . \\
\hline \multicolumn{6}{|c|}{ Intangible } \\
\hline $\begin{array}{l}\text { Tecnologia } \\
\text { Tecnologia unica con } 2 \text { anos } \\
\text { de ventaja en el mercado. }\end{array}$ & si & si & si & $\mathrm{si}$ & si \\
\hline $\begin{array}{l}\text { Reputacion } \\
\text { Posicionamiento fuerte como } \\
\text { servicio unico en el mercado. }\end{array}$ & si & si & si & si & si \\
\hline $\begin{array}{l}\text { Recursos Humanos } \\
\text { Personal cualifcado con años } \\
\text { de experiencia en la empresa }\end{array}$ & $\mathrm{Si}$ & No & . & . & . \\
\hline
\end{tabular}

\section{Análisis DAFO}

Se considera que una de las principales fortalezas del modelo a estudiar se debe a su naturaleza de Software-as-aService basado en la nube, lo que implica que no requiere de una infraestructura física para ser implementado en los sistemas corporativos. Esto significa que el modelo de empresa no requerirá de un establecimiento físico para operar en mercados internacionales, no inicialmente.

Tabla 2. Análisis DAFO. Fuente: propia.

\begin{tabular}{|c|c|}
\hline Debilidades & Amenazas \\
\hline $\begin{array}{l}\text { Falta de experiencia y conocimiento específico } \\
\text { del mercado latinoamericano; } \\
\text { Diferencias culturales que pueden impactar el } \\
\text { desarrollo de negocio; } \\
\text { La falta de presencia física implica un desarrollo } \\
\text { más lento del mercado e integración técnica } \\
\text { más difícil; } \\
\text { Ausencia de benchmark de éxito de } \\
\text { internacionalización previo; }\end{array}$ & $\begin{array}{l}\text { Competidores con productos de función similar, } \\
\text { de bajo precio y con amplio portfolio de } \\
\text { servicios; } \\
\text { La mayoría de los call centers ya disponen de } \\
\text { infraestructura de grabación de llamadas; } \\
\text { Falta de regulación local que implique la } \\
\text { grabación obligatoria de llamadas. }\end{array}$ \\
\hline Fortalezas & Oportunidades \\
\hline $\begin{array}{l}\text { Facilidad de integración que no implica la } \\
\text { inversión en establecimientos físicos o } \\
\text { infraestructura on-premise; } \\
\text { Grabación de llamadas en líneas fijas y móviles } \\
\text { a través de la integración con telcos; } \\
\text { Tecnología avanzada y más de } 28 \text { años de } \\
\text { experiencia en el mercado español con una } \\
\text { Implantación nacional madura; } \\
\text { Contrato de integración con Telefónica en } \\
\text { España }\end{array}$ & $\begin{array}{l}\text { El posible impacto de MiFID II fuera de Europa, } \\
\text { Incremento de la penetración de los servicios } \\
\text { en la nube a nivel global; } \\
\text { El mercado está en fase de pleno crecimiento y } \\
\text { falta de líder }\end{array}$ \\
\hline
\end{tabular}

\section{Análisis del sector}

El sector de grabación de llamadas aún se encuentra en una fase relativamente temprana de su desarrollo.

Con la penetración alta de los dispositivos móviles a nivel mundial y los avances tecnológicos hacia la adopción de los servicios en la nube, cada vez más empresas buscan soluciones integrales que no requieren de mucha inversión en infraestructura física.

Los servicios de grabación de llamadas han sufrido algunos cambios importantes en las últimas décadas. Por una parte, destaca la migración de hardware a soluciones en la nube; además, los dispositivos de grabación físicos suelen ser más lentos, caros e inconsistentes, provocando que cada vez menos empresas inviertan en este tipo de infraestructuras para grabar las llamadas.

En los años 90, las compañías empezaron a vender sofware-as-a-Service directamente a través de internet, lo que permitió a los clientes poder pagar por suscripción en vez de licencias.

En 2006, Amazon introdujo el primer servicio público de computación en la nube [7], a raíz de esto muchas empresas comenzaron a migrar hacia la nube para ofrecer soluciones eficientes.

Tabla 3. Audio Communications Monitoring Market. Fuente: propia a partir de Markets\&Markets, AbNewsWire y Zeon Markert Research.

\begin{tabular}{|c|c|}
\hline & \multicolumn{1}{|c|}{ Audio Communications Monitoring Market } \\
\hline Segmentos & $\begin{array}{r}\text { - Reporting \& Analytics } \\
\text { - Call Recording Software \& Quality Analysis } \\
\text { - Audio Loudness } \\
\text { - Metering \& Monitoring Solution }\end{array}$ \\
\hline Professional Services \\
\hline Tamaño & Support \& Maintenance
\end{tabular}

\section{Mercado de grabación “Audio Communications Monitoring Market"}

Los servicios de grabación de llamadas pertenecen al sector de grabación de llamadas conocido como “Audio Communications Monitoring Market”[8], de sus características destacan, tabla 3:

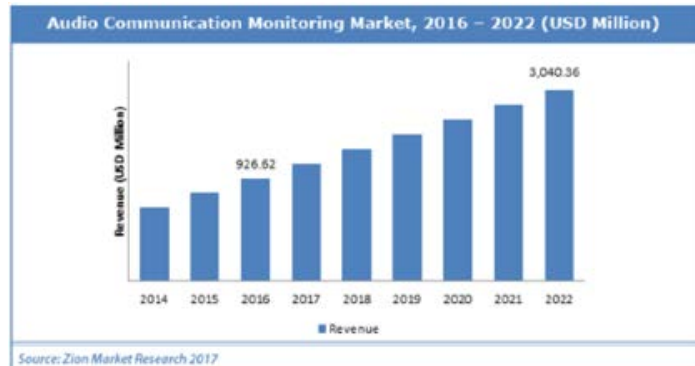

Fig. 1. Audio Communications Monitoring Market. Fuente: Zion Market Rearch 2017. 


\section{Regulaciones de seguridad}

En enero de 2018, la Comisión Europea implementó MiFID II [3], una directiva que impuso regulaciones estrictas en el mercado financiero con el objetivo de mantenerlo transparente y seguro para los inversores. Según estas regulaciones, las empresas que proporcionan servicios financieros están obligadas a grabas todas las llamadas que pueden llevar a una transacción o venta; además, estas grabaciones deben ser almacenadas durante 5 años o incluso 7 años en algunos casos específicos. Como resultado la capacidad para el modelo de empresa de grabar interacciones y almacenar todos los datos en la nube por más de 5 años abrió un mercado importante en Europa.

Como respuesta a la crisis financiera de 2007 y 2008, en 2010 en los Estados Unidos se implementó el DoodFrank Act [9], una directiva con objetivo y regulaciones muy similares a las de MiFID II.

Según el Dood-Frank Act [9], las empresas en el sector financiero tienen que grabar todas las interacciones que lleven a una transacción y mantener las grabaciones durante 5 años; además, las grabaciones deben ser de fácil acceso en la sede de la compañía.

Estas regulaciones son de suma importancia a la hora de evaluar la entrada en el mercado latinoamericano, debido a que ambas tendrán un gran impacto. En el caso de MiFID II, su impacto se extenderá fuera de Europa para todas las empresas que gestionan datos de ciudadanos europeos. Para el caso del Dodd-Frank Act, la directiva norteamericana también tendrá efecto en el mercado latinoamericano para todas las empresas que mantienen relaciones estrechas con compañías o inversores norteamericanos que pueden llevar a transacciones potenciales.

En el caso de MiFID II, hace referencia a todas aquellas empresas de la competencia que operan en varios mercados globales y podrían ofrecer servicio en México, pero no necesariamente tienen un enfoque o presencia fuerte en este país. Para Dodd-Frank Act, esta referencia sería la competencia local en México cuya actividad estaría orientada exclusivamente a las empresas en su territorio, lo que supondría en cierto sentido una competencia mucho más directa para el modelo de empresa a estudiar.

\section{Estudio 1: Competencia Internacional}

Para llevar a cabo el análisis de competencia internacional, se estudiaron alrededor de 10 empresas (Cognia, Aurall, Telstra, Phone Wagon y Atmos, entre otras), de las cuales se seleccionaron las 3 principales que representaran una mayor amenaza para el modelo a estudiar. Entre los criterios para descartar el resto de las empresas destacaron las funcionalidades (demasiado limitadas), el modelo de negocio (competencia no directa) y el precio (muy elevado).

Las empresas que supondría mayor amenaza serian Smarsh, Crazy Call y Cloud Call. Estudio mostrado en la tabla 4.

Tabla 4. Competencia internacional. Fuente: propia a partir de webs oficinales de Smarsh, Crazy Call y Cloud Call.

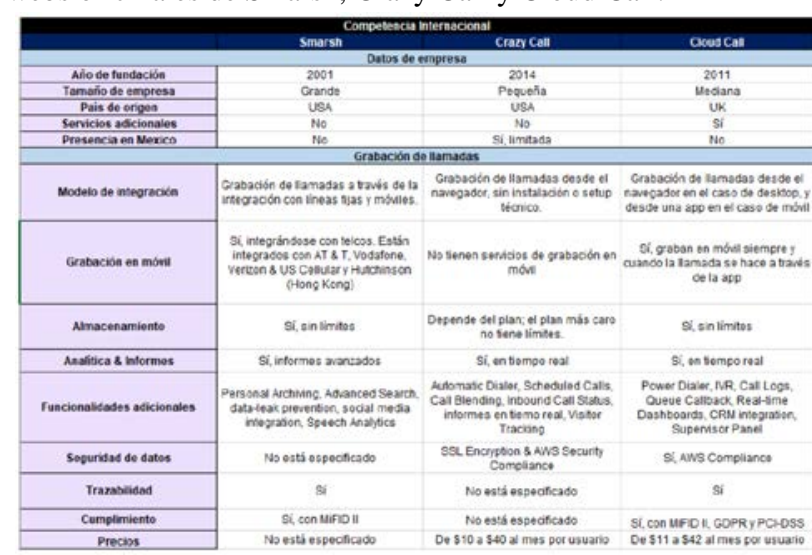

\section{Estudio 2: Competencia Local}

El siguiente análisis conlleva la situación de la competencia local, para ello se estudiaron 5 empresas: SíGraba, TelePROM, Quarea, Jive Communications y Could Call México, la última diferente a la empresa Cloud Call mencionada en el primer estudio.

De las 5 empresas, TelePROM fue excluida por estar enfocada exclusivamente a los servicios de grabación onpremise.

Tabla 5. Competencia Local (México). Fuente: propia a partir de webs oficinales de SíGraba, Jive Communications y Cloud Call México.

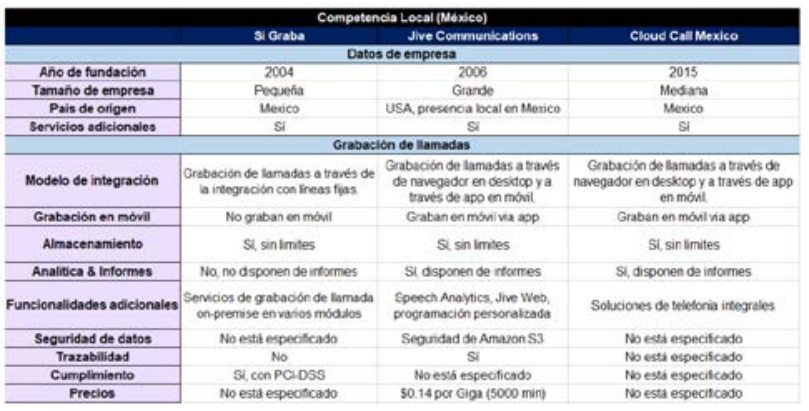

\section{Selección de mercado objetivo}

Una vez establecida el área geográfica de estudio de acuerdo a la localidad del modelo de empresa, se valorarán todos los países que engloba LATAM a través de variables que se consideran significativas para determinar cuales son los 10 países con mejor puntuación, según criterio propio de importancia.

En la tabla 5 se muestran aquellos países excluidos debido a sus condiciones económicas actuales o por no existir información suficiente para su valoración.

Tabla 5. Países excluidos del estudio. Fuente: datos extraídos de [10]. 


\begin{tabular}{|l|c|c|c|c|c|}
\hline \multicolumn{1}{|c|}{ PAis } & $\begin{array}{c}\text { PIB per } \\
\text { cápita 2017 } \\
\text { (int. Dollars) }\end{array}$ & $\begin{array}{c}\text { Crecimiento } \\
\text { real GDP (\% } \\
\text { change) }\end{array}$ & $\begin{array}{c}\text { Doing } \\
\text { business } \\
(\mathbf{1} / 15)\end{array}$ & $\begin{array}{c}\text { Riesgo Pais } \\
\text { (A-E) }\end{array}$ & $\begin{array}{c}\text { Importaciones } \\
\text { servicios 2016 } \\
\text { (US dollars) }\end{array}$ \\
\hline Cuba & $\mathrm{n} / \mathrm{d}$ & $\mathrm{n} / \mathrm{d}$ & $\mathrm{n} / \mathrm{d}$ & $\mathrm{E}$ & $\mathrm{n} / \mathrm{d}$ \\
\hline Guayana Francesa & $\mathrm{n} / \mathrm{d}$ & $\mathrm{n} / \mathrm{d}$ & $\mathrm{n} / \mathrm{d}$ & $\mathrm{n} / \mathrm{d}$ & $\mathrm{n} / \mathrm{d}$ \\
\hline Puerto Rico & $\mathrm{n} / \mathrm{d}$ & $-7,7$ & 12 & $\mathrm{n} / \mathrm{d}$ & $\mathrm{n} / \mathrm{d}$ \\
\hline Venezuela & 11.290 & -14 & $\mathrm{n} / \mathrm{d}$ & $\mathrm{E}$ & $\mathrm{n} / \mathrm{d}$ \\
\hline
\end{tabular}

1. Selección de países

Para la selección de país se considera que la variable fundamental para la internacionalización de un servicio es el tamaño de mercado, en la tabla 6 se muestran la suma de las líneas fijas más móviles del año 2016 [11].

Tabla 6. Tamaño de mercado (líneas fijas y móviles). Fuente: datos obtenidos de la IUT.

\begin{tabular}{|l|c|c|}
\hline \multicolumn{1}{|c|}{ PAis } & $\begin{array}{c}\text { Tamaño de mercado } \\
\text { (lineas Fijas } \\
\text { Móviles) }\end{array}$ & $\begin{array}{c}\text { Paises } \\
\text { seleccionados }\end{array}$ \\
\hline Argentina & 73.662 .322 & $\mathbf{X}$ \\
\hline Brazil & 285.909 .589 & $\mathbf{X}$ \\
\hline Chile & 26.677 .640 & $\mathbf{X}$ \\
\hline Colombia & 65.800 .908 & $\mathbf{X}$ \\
\hline Costa Rica & 9.180 .490 & $\mathbf{X}$ \\
\hline Dominican Republic & $10.075 .000 *$ & $\mathbf{X}$ \\
\hline Honduras & 8.275 .731 & $\mathbf{X}$ \\
\hline Mexico & 132.182 .443 & $\mathbf{X}$ \\
\hline Panama & 5.783 .456 & $\mathbf{X}$ \\
\hline Peru & 40.007 .625 & $\mathbf{X}$ \\
\hline Uruguay & 6.230 .302 & $\mathbf{X}$ \\
\hline
\end{tabular}

Por los datos mostrados en la tabla 6, los dos países con mayor tamaño de mercado son Brasil y México, representando más de la mitad del total de líneas de los países estudiados.
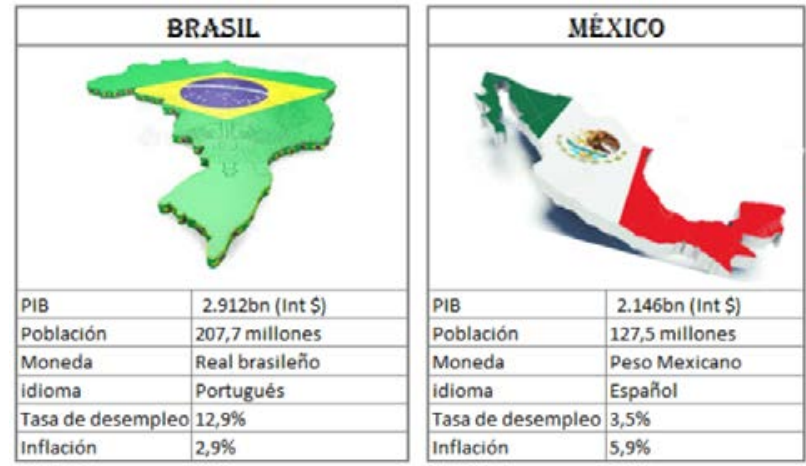

Fig.2. Entorno Macro y Micro. Fuente elaboración propia.

En la figura 2 se muestran los datos económicos de los países seleccionados, se observa que el PIB de Brasil es ligeramente superior al de México, pero teniendo en cuenta que la diferencia en población es mayor que la que existe en el tamaño del PIB, el PIB per cápita es mayor en México. Se destaca que la diferencia en la tasa de desempleo es de un $9,4 \%$ a favor del país mexicano. Con respecto a la tasa de inflación mexicana, 5,9\% se considera elevada y es debido a la volatilidad del precio del combustible. Las autoridades mexicanas han llevado a cabo medidas de estabilización como la subida de los tipos de interés así como la venta de bonos para drenar la liquidez [12].

2. Tamaño de mercado (total de número de líneas fijas y móviles)

En la tabla 7 el tamaño de mercado en términos absolutos de Brasil es mas del doble que el de México, como se ha mencionado antes la población de Brasil es muy superior a la de México; por tanto, se ha dividido el número total de líneas fijas y móviles entre la población de cada uno de los países para poder, en términos relativos, conocer cuál es el tamaño de mercado.

Tabla 7. Número de líneas, móviles+fijos 2016. Fuente: [11]

\begin{tabular}{|l|c|c|}
\cline { 2 - 3 } \multicolumn{1}{c|}{} & Brasil & Mexico \\
\hline № líneas (movil + fijos) 2016 & 285.909 .589 & 132.182 .443 \\
\hline №líneas Total/población & 1,38 & 1,04 \\
\hline
\end{tabular}

3. Cloud computing services

En la tabla 8 se muestra según el estudio BSA Global Cloud Computing Scorecard del 2018 [13], México ocupando el puesto 13 mientras que Brasil se encuentra en el 18, de un total de 24. Este estudio tiene en cuenta las regulaciones y medidas adoptadas por los diferentes países.

Tabla 8. Ranking 24 países. Fuente: [13]

\begin{tabular}{|l|c|c|}
\cline { 2 - 3 } \multicolumn{1}{c|}{} & \multicolumn{2}{c|}{$\begin{array}{c}\text { Posición Ranking } \\
\text { ( } x \text { de 24) }\end{array}$} \\
\cline { 2 - 3 } \multicolumn{1}{c|}{} & Brasil & Mexico \\
\hline Data Privacy & 23 & 2 \\
\hline Security & 14 & 18 \\
\hline Cybercrime & 11 & 11 \\
\hline Intellectual Property & 16 & 16 \\
\hline $\begin{array}{l}\text { Support for Industry- } \\
\text { Led Standards and } \\
\text { International }\end{array}$ & 22 & 7 \\
\hline Promoting Free Trade & 20 & 11 \\
\hline $\begin{array}{l}\text { IT Readiness and } \\
\text { Broadband Deployment }\end{array}$ & 16 & 21 \\
\hline
\end{tabular}

4. Regulaciones del sector de telecomunicaciones y de seguridad

En cuanto a las regulaciones de seguridad MiFID II y el Dodd-Frank Act, su impacto se extenderá al mercado latinoamericano; a pesar de que su implementación no abarque América Latina en sí, todas las empresas en el sector financiero mexicano que gestionan datos de ciudadanos e inversores europeos o norteamericanos se verán afectadas por las directivas. Por otra parte, la reforma de la Ley Federal de Telecomunicaciones y Radiodifusión de México [14], incluye artículos que pueden presentar mas oportunidades para el modelo de 
empresa. De acuerdo al articulo 189 y 190, los operadores telefónicos serán obligados a guardar os metadatos de los usuarios durante un plazo de dos años para posibles consultas por las autoridades correspondientes.

Como no se han encontrado datos disponibles de uso libre para poder cuantificar el volumen de transacciones financieras entre Europa y/o Estados unidos con Brasil y México, se muestra en la tabla 9 una aproximación con datos de Inversión Extranjera Directa con el detalle de los países que más invierten y en qué sectores para nuestra comparativa.

Tabla 9. Comparación entre Brasil y México del volumen de transacciones financieras.

\begin{tabular}{|c|c|c|}
\hline & BRASIL & MÉXICO \\
\hline Situación económica actual & $x$ & $\checkmark$ \\
\hline $\begin{array}{l}\text { Tamaño de mercado } \\
\text { (líneas fijas y móviles) }\end{array}$ & $\checkmark$ & $x$ \\
\hline Cloud Computing Services & $x$ & $\checkmark$ \\
\hline Inversión Extranjera Directa (FDI) & $x$ & $\checkmark$ \\
\hline $\begin{array}{l}\text { Regulaciones Sector } \\
\text { Telecomunicaciones y Seguridad }\end{array}$ & $x$ & $\checkmark$ \\
\hline
\end{tabular}

El país con mayor potencial entonces en LATAM para el modelo de empres es México. De todas las variables analizadas en este apartado, México obtiene mejor valoración en términos generales, por ende, es el país mas optimo para la internacionalización del modelo de empresa a nuevos mercados.

\section{Forma de entrada al mercado}

Para determinar la forma de entrada al mercado de México, previamente se hará un análisis a partir de las ventajas competitivas identificadas y estimación del mercado de grabación de llamadas, la estimación se muestra en la tabla 10.

Tabla 10. Mercado de grabación de llamadas. Fuente: [4]

\begin{tabular}{|l|r|c|c|c|}
\hline \multicolumn{5}{|c|}{ MERCADO DE GRABACIÓN DE LLAMADAS } \\
\hline \multicolumn{1}{|c|}{ País } & $\begin{array}{c}\text { PIB MM \$ } \\
\mathbf{2 0 1 7}\end{array}$ & Peso & $\begin{array}{c}\text { TAMAÑO M\$ } \\
\mathbf{2 0 1 7}\end{array}$ & $\begin{array}{c}\text { Potencial } \\
\text { M\$ }\end{array}$ \\
\hline China & 23.120 & $18,23 \%$ & 251,5 & 554,3 \\
\hline Estados Unidos & 19.360 & $15,27 \%$ & 210,6 & 464,2 \\
\hline India & 9.447 & $7,45 \%$ & 102,8 & 226,5 \\
\hline Japón & 5.405 & $4,26 \%$ & 58,8 & 129,6 \\
\hline Alemania & 4.150 & $3,27 \%$ & 45,1 & 99,5 \\
\hline Rusia & 4.000 & $3,15 \%$ & 43,5 & 95,9 \\
\hline Indonesia & 3.243 & $2,56 \%$ & 35,3 & 77,8 \\
\hline Brasil & 3.219 & $2,54 \%$ & 35,0 & 77,2 \\
\hline Reino Unido & 2.880 & $2,27 \%$ & 31,3 & 69,0 \\
\hline Francia & 2.826 & $2,23 \%$ & 30,7 & 67,8 \\
\hline México & 2.406 & $1,90 \%$ & 26,2 & 57,7 \\
\hline Italia & 2.307 & $1,82 \%$ & 25,1 & 55,3 \\
\hline Turquía & 2.133 & $1,68 \%$ & 23,2 & 51,1 \\
\hline Corea del Sur & 2.027 & $1,60 \%$ & 22,1 & 48,6 \\
\hline Arabia Saudi & 1.789 & $1,41 \%$ & 19,5 & 42,9 \\
\hline España & 1.769 & $1,40 \%$ & 19,2 & 42,4 \\
\hline Canadá & 1.764 & $1,39 \%$ & 19,2 & 42,3 \\
\hline Resto del Mundo & 34.968 & $27,57 \%$ & 380,4 & 838,4 \\
\hline Total Mundial & $\mathbf{1 2 6 . 8 1 3}$ & $\mathbf{1 0 0 , 0 0 \%}$ & $\mathbf{1 3 7 9 , 6}$ & $\mathbf{3 0 4 0 , 4}$ \\
\hline
\end{tabular}

Dada la naturaleza del servicio, se han descartado otras formas de exportación directa como el Comercio Electrónico de Exportación, ya que no es un producto que pueda ser vendido en unidades de manera individual, sino que se trata de un software que se integra a los sistemas de los clientes [19].

Tabla 11. Método de entrada ideal. Fuente: propia.

\begin{tabular}{|c|c|c|c|}
\hline & Situación & Necesidad & $\begin{array}{l}\text { Método de } \\
\text { entrada ideal }\end{array}$ \\
\hline \multirow{2}{*}{$\begin{array}{l}\text { Modelo } \\
\text { de Empresa }\end{array}$} & $\begin{array}{l}\text { Herramienta } \\
\text { Tecnológica }\end{array}$ & $\begin{array}{l}\text { Alto } \\
\text { tecnológico }\end{array}$ & Venta Directa \\
\hline & Ventaja competitiva & 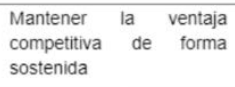 & $\begin{array}{l}\text { Evitar traspaso de } \\
\text { tecnologia }\end{array}$ \\
\hline \multirow{3}{*}{$\begin{array}{l}\text { Mercado } \\
\text { Grabación } \\
\text { de llamadas } \\
\text { en México }\end{array}$} & $\begin{array}{l}\text { Potencial } \\
\$ 30,4 \mathrm{M}\end{array}$ & $\begin{array}{l}\text { Alta implicación comercial } \\
\text { y dedicación a la } \\
\text { penetración de mercado }\end{array}$ & $\begin{array}{l}\text { Alto control de } \\
\text { atención al cliente }\end{array}$ \\
\hline & $\begin{array}{l}\text { Tamaño actual } \\
13,7 \mathrm{MS}\end{array}$ & $\begin{array}{l}\text { Sin ahogar el proyecto } \\
\text { inicialmente }\end{array}$ & Costes bajos \\
\hline & $\begin{array}{l}\text { Crecimiento del } \\
\text { mercado } \\
22,02 \% \text { GACR }\end{array}$ & $\begin{array}{l}\text { Costes variables sobre } \\
\text { ventas. Motivador para } \\
\text { agentes y distribuidores }\end{array}$ & $\begin{array}{l}\text { Principalmente } \\
\text { costes variables }\end{array}$ \\
\hline
\end{tabular}

Tabla 12. Método de entrada ideal. Fuente: propia.

\begin{tabular}{|c|c|c|c|c|c|}
\hline Modos de entrada & Venta directa & $\begin{array}{l}\text { Control de la } \\
\text { atención al cliente }\end{array}$ & Coste & $\begin{array}{l}\text { Principal tipo de } \\
\text { coste }\end{array}$ & $\begin{array}{l}\text { Riesgo copia } \\
\text { de la patente }\end{array}$ \\
\hline Método ideal: & si & Alto & Bajo & Variable & Bajo \\
\hline Agente (Elegido) & si & Medio & Bajo & Variables & Bajo \\
\hline Distribuidor & No & Bajo & Bajo & Variables & Bajo \\
\hline Filial & si & Alto & Alto & Fijos & Bajo \\
\hline Delegación & si & Alto & Alto & Fijos & Bajo \\
\hline Strategic Al. & No & Bajo & Medio & Ambos & Alto \\
\hline
\end{tabular}

1. Distribuidores potenciales 
El objetivo serán las tres principales empresas Telefónicas de México que representan el 99\% de las líneas

móviles del país. En la tabla 13 se muestran las tres más grandes empresas de telefonía de México, ordenadas por prioridad de integración.

Tabla 13. Empresas de telefonía móvil en México. Fuente: propia.

\begin{tabular}{|c|c|c|l|}
\hline TELCO & $\begin{array}{c}\text { Usuarios } \\
\text { (Millones) }\end{array}$ & $\begin{array}{c}\text { Cuota de } \\
\text { Mercado } \\
\text { (\% usuarios) }\end{array}$ & \multicolumn{1}{|c|}{ Prioridad } \\
\hline $\begin{array}{c}\text { Telefónica } \\
\text { México }\end{array}$ & 25,8 & $23 \%$ & $\begin{array}{l}\text { Prioridad 1.Mayor facilidad de entrada. } \\
\text { Recordia ya está integrado en España } \\
\text { desde 2017 }\end{array}$ \\
\hline $\begin{array}{c}\text { American } \\
\text { Mobile }\end{array}$ & 73,3 & $65 \%$ & $\begin{array}{l}\text { Prioridad 2. Compañía con más cuota } \\
\text { de mercado mexicano }\end{array}$ \\
\hline AT\&T & 12,5 & $11 \%$ & $\begin{array}{l}\text { Prioridad 3. Tercera en cuota y una de } \\
\text { las más grandes del mundo }\end{array}$ \\
\hline $\begin{array}{c}\text { Operadores } \\
\text { Virtuales }\end{array}$ & 1,1 & $1 \%$ & Escasa Relevancia \\
\hline
\end{tabular}

2. Cadena de valor

La figura 3 resume la cadena de valor para la entrada en el mercado México. En España se mantienen las funciones tecnológicas y estratégicas de marketing, manteniendo una exportación directa.

Neoris sirve de intermediario tecnológico con las empresas de telecomunicaciones de México, aportando la labor comercial consultiva y relacional.

Las empresas de telecomunicaciones distribuyen las soluciones del modelo de empresa entre sus empresas clientes. Aportan la atención al cliente, servicio y garantía, además de ser responsables de establecer el precio final.

El sector BFSI (Banking, Financial, Service \& Insurance) es el consumidor final del modelo de empresa, usando la solución de forma relacional con clientes, partners y proveedores.

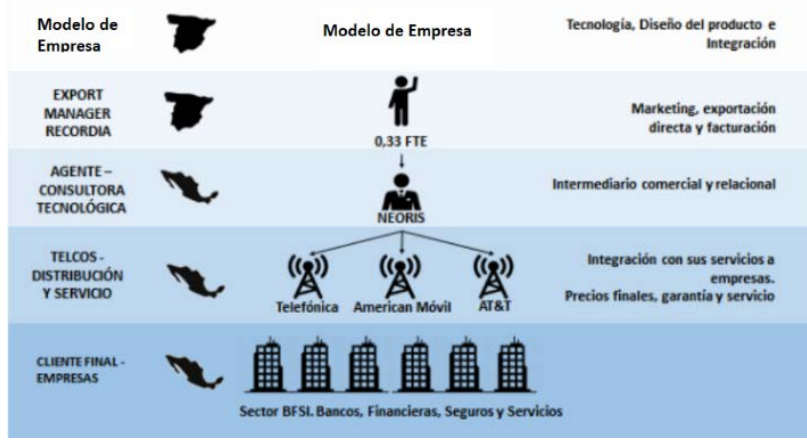

Fig.3. Cadena de valor. Fuente: propia.

\section{Plan de marketing internacional}

Para establecer un sector objetivo, se considera el potencial de mercado creado por las nuevas regulaciones en seguridad. El alcance de la normativa implica a todas las empresas que realicen transacciones con Europa y Norteamérica, considerando que todas las empresas en el sector financiero mexicano que gestionan datos de ciudadanos e inversores europeos o norteamericanos se verán afectadas por estas directivas.
A partir de los datos de población activa en "Servicios profesionales, financieros y comerciales” [15] en 2018 se establece un potencial de mercado en unidades de líneas de 935.189, mostrada en la tabla 14.

Tabla 14. Ocupación 2018 INEGI Encuesta Nacional de Ocupación y Empleo según sector de actividad económica, nacional trimestral. Fuente: INEGI y LinkedIn.

\begin{tabular}{|c|c|}
\hline $\begin{array}{c}\text { Ocupación } 2018 \text { INEGI. Encuesta Nacional de Ocupación y Empleo } \\
\text { Población ocupada según sector de actividad económica, nacional trimestral }\end{array}$ \\
\hline $\begin{array}{c}\text { Servicios profesionales, financieros y } \\
\text { corporativos (INEGI, 2018) }\end{array}$ & 3740759 empleados \\
\hline Puestos comerciales (Fuente Linkedin) & $25 \%$ \\
\hline Potencial mercado en Unidades & 935.189 Líneas \\
\hline
\end{tabular}

1. Objetivos cualitativos

- Establecer notoriedad de marca y generar visibilidad en el mercado

- Posicionar el servicio como un producto único en el mercado

- Posicionar al modelo de empresa como empresa innovadora en el desarrollo de servicios en la nube

- Establecer relaciones a largo plazo con los distribuidores (las empresas de comunicación).

2. Objetivos cuantitativos

- Generar ventas de 72.000,00 euros el primer año y $159.608,00$ el quinto

- Aumentar el numero de seguidores en LinkedIn en un $15 \%$

- Obtener por lo menos 30 conversaciones en el primer mes de publicidad en AdWords, con un incremento de $5 \%$ en cada mes siguiente.

3. Marketing digital

La estrategia de Marketing Digital se llevará a cabo por los siguientes canales principales:

\section{Search Engine Optimization}

En el caso de SEO, o el posicionamiento orgánico en los motores de búsqueda, no habrá inversión directa ya que la estrategia en sí implica que el posicionamiento sea natural sin necesidad de pago. Por otra parte, considerando que no se hará página web especial para México, la publicación de artículos y el posicionamiento orgánico en Google se llevaran por el equipo de Marketing en España.

\section{Social Media}

Las redes sociales están directamente vinculadas con el posicionamiento orgánico de la empresa. Como resultado, la publicación de los posts también se llevará a cabo por el equipo de España [18]. Es importante especificar que no se hará inversión en publicidad en redes sociales por las siguientes razones:

- Facebook, es una herramienta poco relevante para el sector B2B en el que se encuentra el modelo de empresa. La publicidad en Facebook es económica, pero no suele generar conversiones 
en el sector B2B y por ende no se llevará a cabo esta inversión.

- Twitter, la gestión de la cuenta en Twitter la lleva el equipo de marketing en España y no se va a crear una cuenta separada para México.

- LinkedIn, es la herramienta ideal para el sector $\mathrm{B} 2 \mathrm{~B}$, pero los anuncios tienen un coste muy elevado y no necesariamente generan conversiones. En un futuro se puede contemplar una estrategia, pero por el momento se estima que la inversión no sería eficiente.

- Google +, no dispone de red de publicidad.

- E-mail Marketing, el envío del Newsletter a clientes actuales y potenciales se seguirá haciendo de la forma habitual que maneja el modelo de empresa, frecuencia de una vez por semana.

- Search Engine Marketing, el presupuesto destinado a la estrategia de marketing digital (70\% del presupuesto entero) consistirá en una inversión en AdWords, considerando que los Display Ads relevantes para un producto SaaS; se desarrollarán anuncios de texto para Google Search Network.

Se van a crear dos Landing pages: una destinada al sectos BFSI y una genérica para el resto de los clientes potenciales. Para cara Landing Page, se van a elaborar anuncios específicos para captar una cuota de mercado en México, con sus correspondientes palabras claves. En la figura 4,5 y 6 se muestran anuncios y palabras claves seleccionadas para llevar a cabo la estrategia.

Landing Page: Genérica

Palabra Clave: Grabador de llamadas

Volumen: 1,000

\section{Grabador de llamadas telefónicas - Líneas fiias y móviles}

Ad www.cws.com/Grabación-llamadas

Graba las llamadas de tu empresa en lineas fijas y móviles. $99.9 \%$ uptime

Grabador de llamadas México - Para Grandes Empresas

Ad WWW. cWs. com/Grabación-llamadas

Graba llamadas telefónicas en lineas fijas y móviles. Paga sólo por lo que utilizas.

Graba conversaciones en la nube - Grabador de llamadas México

Ad www.cws.com/Grabación-llamadas

Grabación de llamadas. Almacenamiento en la nube. 99.9\% uptime

Fig.4. Landing Page, palabras claves.

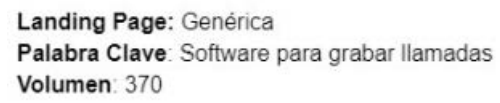

Graba llamadas en la nube - Software para arabar llamadas

Ad WWW.cWs.com/Grabación-llamadas

Graba llamadas y almacena datos en la nube. Paga solo por lo que utilizas.

Software para grabar llamadas - Líneas filas y móviles México Ad WwW.cWs. com/Grabación-llamadas

Grabación de llamadas profesional en la nube. Para empresas medianas y grandes

Fig.5. Landing Page, palabras claves.

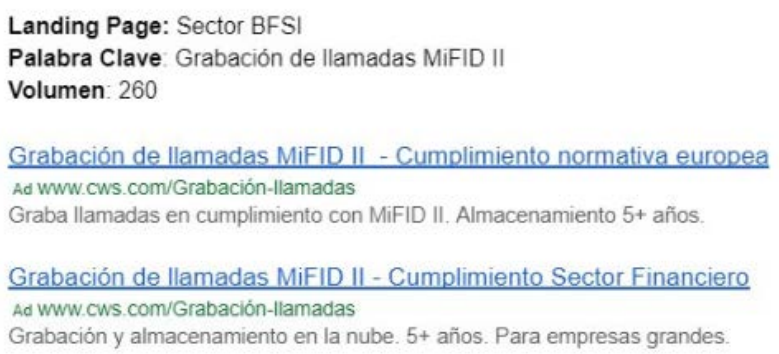

Fig.6. Landing Page, palabras claves.

\section{Plan Económico}

El plan económico-financiero a presentar será una estimación del modelo de empresa con el objetivo de tomar la decisión más importante de todas: si es viable o no la internacionalización del modelo de empresa a México. [16]

La tabla 15 muestra el análisis de solvencia del modelo de empresa; la rentabilidad económica (ROA) en el 2016 alcanzo un nivel positivo del 1\%. El aumento generado entre el período 2014 y 2015 se ha debido a la rotación de activos, generando en la rentabilidad económica un incremento del $9 \%$ y en la rentabilidad financiera del 12\%. El resultado de este análisis ha generado un fondo de maniobra positivo e igual a 1.277.645,03 euros, lo que equivale una proporción del $60,88 \%$ del activo total de la empresa. La mejora que se observa entre 2015-2016 ha sido generada principalmente por una disminución del pasivo corriente.

Tabla 15. Análisis de solvencia del modelo de empresa. Fuente: propia.

\begin{tabular}{|c|c|c|c|c|c|}
\hline \multicolumn{6}{|c|}{ ANAIIISIS DF SOIVENCIA } \\
\hline RATIOS & $31 / 12 / 2016$ & $31 / 12 / 2015$ & $31 / 12 / 2 / 2014$ & $31 / 12 / 2013$ & RATIOSECTORIALI \\
\hline 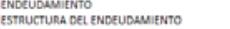 & 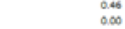 & $\begin{array}{c}a_{0}, 1 \\
\infty \infty \infty\end{array}$ & $\begin{array}{l}1306 \\
0.00\end{array}$ & $\begin{array}{l}1.07 \\
0.00\end{array}$ & $\begin{array}{l}1210 \\
0.00\end{array}$ \\
\hline Fonso se Maniobra & 1.277 .645 .03 & $1.227,499.93$ & $1075,897.09$ & $1.000,57900007>0$ & \\
\hline 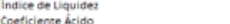 & 292 & 227 & 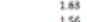 & 2.188 & 2.28 \\
\hline Indice de Tessertio & 000 & 101 & 0.47 & 0.28 & \\
\hline Periedo medio de venta del att: & & & & 6.18 & \\
\hline 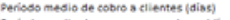 & 121.8 & 122,12 & $160 \%$ & 18908 & \\
\hline 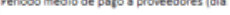 & & & & & \\
\hline man & 16483 & $105 n$ & 21586 & 23,255 & \\
\hline
\end{tabular}

1. Análisis financiero

En análisis financiero va enfocado en estimaciones de costes y análisis de los servicios prestados por el modelo de empresa, estos datos son mostrados en la tabla 16.

Tabla 16. Estimación de costes para plan financiero. Fuente: propia. 


\begin{tabular}{|c|c|c|c|}
\hline INVERSIONES & $\frac{\text { Coste }}{1,500.00}$ & Vida útil & $\begin{array}{l}\text { Amortización Anual } \\
500.00\end{array}$ \\
\hline TOTAL & $1,500.00$ & TOTAL & 500.00 \\
\hline $\begin{array}{l}\text { Tesorería inicial } \\
\text { FINANCIACIÓN }\end{array}$ & $8,000.00$ & & \\
\hline HINAINCIACIOIN & $20,000.00$ & & \\
\hline Deuda bancos & . & & \\
\hline Ventas & $\frac{\text { aก̃o } 1}{108000}$ & Crecimiento olobal del sector & $2202 \%$ \\
\hline Precio por ventas mayores a 100 & 1,080.00 & & \\
\hline $\begin{array}{l}\text { Unidades } \\
\text { Unesd }\end{array}$ & $1,800.00$ & & \\
\hline $\begin{array}{l}\text { Precio por Ventas menores a } 100 \\
\text { servicis/empresa }\end{array}$ & 28.00 & & \\
\hline TOTAL & $72,000.00$ & & \\
\hline Publicidad año 0 & $24,000.00$ & & \\
\hline $\begin{array}{r}\text { Export Manager (0.3 FTE) } \\
\text { OTROS GASTOS }\end{array}$ & $14,560.00$ & Inflación de España 2018 & $1.25 \%$ \\
\hline Comisiones a NEORIS & $5,040.00$ & & \\
\hline Viajes de negocios & $12,000.00$ & & \\
\hline Servicios Amazón Web Service & 4,878.60 & & \\
\hline Mantenimiento de publicidad & $18,000.00$ & & \\
\hline Licencia de Microsoft & 126.00 & & \\
\hline Impuesto España & $250 \%$ & & \\
\hline
\end{tabular}

No se ha considerado una solicitud de préstamo a entidades bancarias u otras entidades financieras debido a la poca inversión inicial que requiere el plan financiero presentado.

Como resultado se ha estipula para el plan financiero $72.000,00 €$, los cuales han venido definidos por un $36,50 \%$ en ventas de líneas a un coste de $20 €$ y un $63,50 \%$ a un coste de $28 €$.

Es importante recalcar que la estimación de almacenamiento en Amazon Wed Service se desarrolla a través del cálculo de volúmenes de Amazon EBS, obteniendo como resultado un total de 348,56€. [17]

En la tabla 17 se muestra el resultado del plan financiero, observándose que el modelo de empresa es lo suficientemente rentable para salir, demostrado con un TIR del 111\%.

Tabla 17. Resultado final de proyección financiera. Fuente: propia.

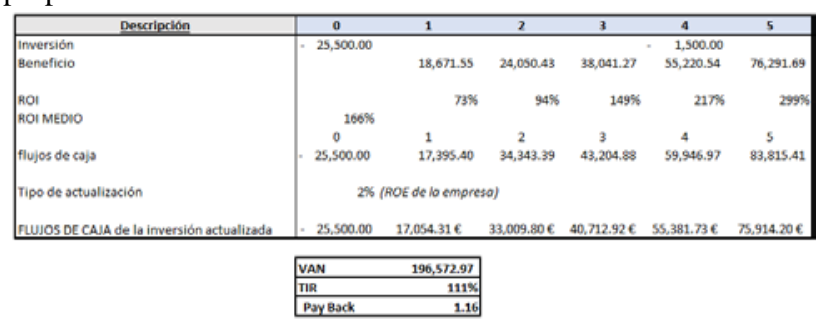

\section{Conclusión}

Se concluye que si es posible llevar a cabo un proceso de internacionalización exitoso en México para el modelo de empresa estudiado apalancando el proyecto fundamentalmente en las alianzas con las empresas telcodistribuidoras y la experiencia de implantación exitosa que ha tenido el modelo estudiado. Así mismo, el proyecto no supone un coste importante para el modelo, ya que la inversión inicial es baja.

Es importante destacar que el sector de Audio Communications monitoring Market se encuentra en crecimiento y las proyecciones de crecimiento para la industria en general son muy prometedoras; además, el mercado en México [20] posee poca competencia y una creciente demanda impulsada fundamentalmente por la necesidad de cumplir con las regulaciones de seguridad impuestas en Europa y Estados Unidos.

\section{REFERENCIAS}

[1] Colomo-Palacios R, Casado-Lumbreras C, Soto-Acosta P, García-Crespo Á. Using the Affect Grid to Measure Emotions in Software Requirements Engineering [Internet]. [cited $2019 \mathrm{Feb}$ 27]. Available from: http://jucs.org/jucs_17_9/using_the_affect_grid/jucs_17_09_128 1_1298_colomo.pdf

[2] Forrester JW. Lessons from system dynamics modeling. Syst Dyn Rev [Internet]. 1987 [cited 2019 Feb 28];3(2):136-49. Available from: http://doi.wiley.com/10.1002/sdr.4260030205

[3] Accenture. Markets in Financial Instruments Directive II (MiFID II) | Accenture [Internet]. 2014 [cited 2019 Feb 6]. Available from: https://www.accenture.com/t20180207T082729Z_w___usen/_acnmedia/PDF-71/Accenture-Market-in-FinancialInstruments-Directive.pdf

[4] Market Research Z. Audio Communication Monitoring Market Increasing Rapidly To Cross USD 3.040 Million by 2022 [Internet]. 2018 [cited 2019 Feb 6]. Available from: https://www.zionmarketresearch.com/news/audiocommunication-monitoring-market

[5] Comisión Europea. Reforma de 2018 de las normas de protección de datos de la UE | Comisión Europea [Internet]. [cited 2019 Feb 26]. Available from: https://ec.europa.eu/commission/priorities/justice-andfundamental-rights/data-protection/2018-reform-eu-dataprotection-rules_es

[6] Council SS. Payment Card Industry (PCI) Data Security Standard Requirements and Security Assessment Procedures Document Changes [Internet]. 2016 [cited 2019 Feb 26]. Available from: https://pcicompliance.stanford.edu/sites/g/files/sbiybj7706/f/pci_ dss_v3-2.pdf

[7] AWS AWS. Centros de datos: nuestros centros de datos [Internet]. [cited 2019 Feb 26]. Available from: https://aws.amazon.com/es/compliance/data-center/data-centers/ MarketsandMarkets. Market Research Reports, Marketing Research Company, Business Research by MarketsandMarkets [Internet]. 2018 [cited 2019 Feb 26]. Available from: https://www.marketsandmarkets.com/

[9] CONSUMER B-FWSRA. Dodd-Frank Wall Street Reform and Consumer Protec [Internet]. 2018 [cited 2019 Feb 26]. Available from: https://legcounsel.house.gov/Comps/Dodd-Frank Wall Street Reform and Consumer Protection Act.pdf

[10] Datosmacro.com. Economia Macroeconomia Todos los datos macro | datosmacro.com [Internet]. [cited 2019 Feb 27]. Available from: https://datosmacro.expansion.com/

[11] International Communication Union. UIT [Internet]. [cited 2019 Feb 27]. Available from: https://www.itu.int/es/Pages/default.aspx

[12] International Monetary Fund. Mexico: 2017 Article IV Consultation-Press Release; and Staff Report [Internet]. 2017 [cited 2019 Feb 27]. Available from: https://www.imf.org/en/Publications/CR/Issues/2017/11/10/Mex ico-2017-Article-IV-Consultation-Press-Release-and-StaffReport-45398

[13] BSA. 2018 BSA Global Cloud Computing Scorecard [Internet]. 2018 [cited 2019 Feb 27]. Available from: https://cloudscorecard.bsa.org/2018/

[14] De Diputados C, Congreso De DH, Unión LA, Ley N. LEY FEDERAL DE TELECOMUNICACIONES Y RADIODIFUSIÓN [Internet]. [cited 2019 Feb 27]. Available from:

https://www.ucol.mx/content/cms/13/file/federal/LEY_FED_DE TELECOMUNICACIONES.pdf

[15] INEGI. Instituto Nacional de Estadística y Geografía. INEGI. [cited 2019 Feb 27]; Available from: https://www.inegi.org.mx/ 
[16] Temiño Aguirre, I. El Mercado Hipotecario en Latinoamérica. ESIC Editorial, Madrid, 2007

[17] Temiño Aguirre, I. AMAZON, lectura empresarial. ESIC Editorial, Madrid, 2017

[18] Puebla Sánchez, I. La adopción de las Tecnologías de la Información y Comunicación en los jóvenes. Selección de un Modelo de Referencia para el Análisis, desde una perspectiva multidisciplinar: Ética, Social y Económica. Tesis Doctoral Capítulos V y VI [páginas: 351-435] Universidad Francisco de Vitoria. Madrid 2014. Disponible en TESEO: https://www.bvsspa.es/profesionales/bbdd-y-otros-

recursos/recursos/teseo-base-de-datos-de-tesis-doctorales

[19] Colomo-Palacios R, Gomez-Berbis J.M., García-Crespo A, and Puebla-Sanchez I. Social Global Repository: using semantics and social web in software project. Vol.4, International Journal of Knowledge and Learning. January 2008

[20] Puebla Sanchez MI, Temiño Aguirre I. International Journal of Information Systems and Software Engineering for Big Companies[Elektronische Ressource]]. [Internet]. Vol. 5, International Journal of Information Systems and Software Engineering for Big Companies: IJISEBC, ISSN-e 2387-0184, Vol. 5, No. 2 (Diciembre/December), 2018-2018, págs. 115-122. United academic journals; 2014 [cited 2019 Mar 7]. 115-122 p. Available from: https://dialnet.unirioja.es/servlet/articulo?codigo=6739291 